\title{
Surface functionalization of polymeric nanospheres modulates macrophage activation: relevance in Leishmaniasis therapy
}

Daniela Barros ${ }^{1}$, Sofia A Costa Lima ${ }^{*}, \neq, 1$ \& Anabela Cordeiro-da-Silva ${ }^{*},, 1,2$

${ }^{1}$ IBMC-INEB, Infection \& Immunology Unit - Parasite Disease Group, University of Porto, Rua do Campo Alegre, 823, 4150-180 Porto, Portugal;

${ }^{2}$ Departamento de Ciências Biológicas, Faculdade de Farmácia, Universidade do Porto, Rua Jorge Viterbo Ferreira, 228, 4050-313 Porto, Portugal

*Authors for correspondence: slima@ff.up.pt \& cordeiro@ibmc.up.pt

${ }^{\ddagger}$ Authors contributed equally

Keywords: carbohydrates - immunotherapy • mannose receptors • murine primary macrophages $•$ PLGA nanospheres

Originally published at Nanomedicine (London), 2015, 10(3):387-403, doi: 10.2217/nnm.14.116, Future Science Group 


\section{ABSTRACT}

Aim: To characterize the production and application of carbohydrate functionalized poly $(\mathrm{d}, \mathrm{I}$ lactide-co-glycolide) (PLGA) nanospheres as immune-modulatory mediators in the treatment of visceral leishmaniasis (VL). Materials \& methods: PLGA nanospheres were prepared by nanoprecipitation and surface functionalized with mannose, mannan or mannosamine moieties using a carbodiimide reaction. Flow cytometry and fluorescence microscopy revealed the interaction of these nanospheres with macrophages. Results: The nanocarriers were taken up by murine primary macrophages using clathrin-mediated endocytosis. Co-culture of macrophages with carbohydrate-functionalized nanospheres led to their activation and production of pro-inflammatory cytokines. One dose of amphotericin B-loaded on mannanfunctionalized nanospheres resulted in an efficacy VL therapy. Conclusion: This approach provides a promising therapy for VL and a new therapeutic nanoplatform for obligate intracellular pathogens.

\section{INTRODUCTION}

The application of polymeric nanoparticles as delivery systems is becoming rather popular, as their use promise to overcome several problems associated with the administration of some drugs, vaccines and DNA [1]. The US FDA-approved poly(d,I-lactide-co-glycolide) (PLGA) polymer is widely used in delivery systems, and has gained particular significance due to its biocompatibility, biodegradability and capacity to maintain the drug in therapeutic levels for prolonged periods of time [2]. The polymer matrix protects the drug from degradation and the levels of its release from the nanoparticle can be manipulated by alterations in the formulation or in its surface. PLGA nanoparticles can exhibit a size similar to pathogens and be rapidly and efficiently phagocytized by antigen presenting cells (APCs), such as macrophages and dendritic cells (DCs), eliciting potent humoral and cellular immune response to the incorporated or adsorbed ligand [3]. It has been demonstrated that functionalized PLGA nanoparticles could elicit a potent cytotoxic T-lymphocyte immune response [4], which is perhaps related with the nanoparticle surface modification.

Macrophages play a major role in connecting innate and adaptive immunity, which is important to favor the development of a protective immune response. These cells express a broad range of plasma membrane receptors that mediate their interactions with natural host components, as well as a range of microorganisms, which is crucial to its functions in homeostasis as well as host defense in innate and acquired immunity, autoimmunity, inflammation and immunopathology. C-type lectin receptors (CLRs) are highly conserved pattern recognition receptors with carbohydrate-recognition domains that bind sugars (e.g., mannose [M], fucose and $\mathrm{N}$-acetylglucosamine) in a calcium-dependent manner, and can be found in the surface of many pathogens (bacteria, fungi and virus). In particular, mannose receptor (MR; CD206) was the first reported member of a family of four endocytic CLRs receptors, with a role in clearance of endogenous glycoproteins and in pathogen recognition/antigen presentation. Targeting pattern recognition receptors on macrophage offers the advantage of triggering specific signaling pathways to induce a tailored and robust immune response.

Several studies have demonstrated the effectiveness of targeting CLRs to activate APCs, particularly DCs using mannoproteins from pathogens, by conjugation of glycans directly to the antigen or a carbohydrate to nanoparticles [5]. However, only a few studies have explored the use of carbohydrate-functionalized nanocarriers as a targeting drug delivery system 
toward macrophages [6, 7]. Here, we describe functionalization of PLGA nanospheres with three conserved carbohydrate structures ( $\mathrm{M}$, mannan $[\mathrm{MN}]$ and mannosamine $[\mathrm{Ms}])$ that have high affinity to MR on macrophages. We also investigate the mechanisms by which these functionalized PLGA nanospheres are internalized by and influence the activation of murine primary macrophages. The studies described herein demonstrate that $\mathrm{MN}$-functionalized PLGA nanospheres induce a receptor-mediated activation of macrophages promoting a proinflammatory environment that was further exploited as an immune-therapy approach toward visceral leishmaniasis $(\mathrm{VL})$.

\section{MATERIALS AND METHODS}

Ethics statement. All procedures involving animals were approved by and performed in accordance with the Portuguese National Authority for Animal Health license

$(0042 / 000 / 000 / 2012)$ and the requirements/regulations lay down by the ethical review committee of IBMC. INEB Animal Ethics Committee. Animals were kept in ventilated cages and provided with food and water ad libitum and the 3Rs were respected. SA Costa Lima and A Cordeiro da Silva have an accreditation for animal research given from Portuguese National Authority for Animal Health (Ministerial Directive 1005/92).

Reagents. Poly (vinyl alcohol) (87-89\% hydrolyzed, MW 13,000-23,000 g/mol); D-(+)-M; MN, from Saccharomyces cerevisiae; D-Ms hydrochloride; N-hydroxy-succinimide; N-(3dimethylaminopropyl)-N'-ethyl-carbodiimide hydrochloride (DCC); ethylenediamine dihydrochloride (EDA); amphotericin B (AmB) from Streptomyces; and fluorescein isothiocyanate isomer I (FITC) were all purchased from Sigma-Aldrich (Sintra, Portugal). PLGA [70:30] was acquired from Poly-sciences, Inc. (Warrington, UK). The monoclonal antibodies were obtained from Biolegend (CA, USA). All other chemicals and reagents used in the study were reagent grade or higher.

Preparation of fluorescein-loaded PLGA nanospheres. The FITC-loaded PLGA nanospheres were prepared in aseptic conditions. FITC was used as a model drug and its incorporation into PLGA nanospheres was obtained using the nanoprecipitation method as described [8]. All FITC-nanoformulations were tested for the presence of endotoxin using a kit (CellTox ${ }^{\mathrm{TM}}$; Promega, WI, USA) accordingly to the manufacturer instructions, and were shown to be endotoxin free.

PLGA nanospheres surface functionalization. The functionalization with $\mathrm{M}$ was done by a carbodiimide chemical reaction. Briefly, $153 \mu \mathrm{g} \mathrm{DCC}, 459 \mu \mathrm{g} \mathrm{N}$-hydroxysuccinimide and 160 $\mu \mathrm{g}$ EDA was added to $10 \mathrm{mg}$ of PLGA nanospheres, dispersed in $5 \mathrm{ml}$ acidic phosphate buffered saline (PBS pH 5.0) and the mixture was then stirred at room temperature overnight. To the amine-terminated PLGA nanospheres, previously recovered by centrifugation, $20 \mathrm{mg}$ of $M$ was added and the mixture left under stirring overnight. The $M$ functionalized nanospheres were then recovered by centrifugation. The functionalization with $\mathrm{MN}$ and Ms followed the above description of the carbodiimide chemical reaction without the presence of EDA [9].

Characterization of the nanospheres: size, zeta potential, surface morphology \& carbohydrate content. Functionalized and nonfunctionalized nanospheres were characterized in terms of mean particle size, size distribution and zeta potential using a Zetasizer Nano ZS detector (Malvern Instruments, UK). The nanospheres were diluted with distilled water or culture medium complemented with $10 \%$ fetal bovine serum (FBS) to a final 
concentration of $1 \mathrm{mg} / \mathrm{ml}$ and evaluated immediately or $24 \mathrm{~h}$ after incubation at $37^{\circ} \mathrm{C}$. The morphology was observed by transmission electron microscopy (TEM, Jeol JEM-1400). The aqueous dispersion of nanospheres was placed on a carbon coated copper grids followed by negative staining with uranyl acetate and placed at the accelerating voltage of $60 \mathrm{kV}$. The level of incorporation of $\mathrm{M}$ and $\mathrm{MN}$ in PLGA nanospheres was determined by indirect quantification of the ligand present in the supernatants recovered from the washings of functionalized nanospheres, using phenol-sulfuric acid assay. In the same way, the ninhydrin assay was used to quantify the amount of Ms attached to the nanospheres. The total amount of sugar $(\mu \mathrm{g} / \mathrm{mg})$ was expressed in relation to polymer mass.

Agglutination assay. To verify the activity of the carbohydrate moieties associated to the surface of PLGA nanospheres, an in vitro agglutination assay was performed. The timedependent increase in turbidity at $550 \mathrm{~nm}$ was monitored spectrophotometrically (Sinergy 2, BioTek; Bedfordshire, UK) for $90 \mathrm{~min}$ at $25^{\circ} \mathrm{C}$.

Determination of drug loading \& encapsulation efficiency. The FITC loading was quantified by fluorimetry (Sinergy 2, BioTek; Bedfordshire, UK), after excitation at wavelength of $485 \pm 10 \mathrm{~nm}$ the fluorescence emission was obtained at $528 \pm 10 \mathrm{~nm}$. Linear calibration curves were made in the range of $0.2-50.0 \mu \mathrm{g} / \mathrm{ml}$ and were used to quantify the nonincorporated compound.

FITC in vitro release from the PLGA nanospheres. FITC release from the nonfunctionalized and functionalized PLGA nanospheres was evaluated over time in PBS buffer pH 7.4. A given amount of the nanoformulation correspondent to $0.5 \mathrm{mg}$ of FITC was dispersed in $1.0 \mathrm{ml}$ of PBS pH 7.4 and incubated at $37^{\circ} \mathrm{C}$ with shaking at $500 \mathrm{rpm}$. At known time intervals, nanospheres were separated from the supernatant through centrifugation. The nanospheres were then resuspended in fresh release medium and re-incubated in the same conditions. FITC present in the supernatant was quantified by fluorimetry in a microplate reader, as described above.

Isolation \& culture of murine bone marrow-derived macrophages. Macrophage primary cultures were generated as described previously [10]. For bone marrow-derived macrophages $(\mathrm{BMM}-\varphi)$ differentiation $5 \%$ L-cell conditioned medium was added at days 0 and 4 , and at day 7 half of the culture medium was renewed with $5 \%$ L-cell conditioned medium supplemented DMEM.

Flow cytometric studies. Flow cytometry was done in a FACS Canto II (BD Biosciences, Madrid, Spain) and in all studies at least 10,000 events were analyzed, per sample.

MR expression. The expression of MR was assessed by flow cytometry on different days of BMM- $\varphi$ differentiation. At days 6, 7, 8 and 9, adherent cells were recovered, washed with cold FACS buffer $(0.01 \%$ sodium azide, $2 \%$ FBS and $1 \%$ antibiotics mixture, $100 \mathrm{U} / \mathrm{ml}$ penicillin and $100 \mu \mathrm{g} / \mathrm{ml}$ streptomycin, in PBS $\mathrm{pH} 7.4$ ) and stained with FITC-conjugated antimouseF4/80 and APC-conjugated antimouse-CD206 on ice during $30 \mathrm{~min}$.

Cellular uptake studies. The cellular uptake of FITC-loaded nanospheres was evaluated in ВMM- $\varphi$ at day 8 of differentiation upon incubation with nonfunctionalized and functionalized $(0.5 \mathrm{mg} / \mathrm{ml})$ PLGA nanospheres. After specific times, cells were washed with PBS $\mathrm{pH} 7.4$ to remove noninternalized nanospheres and recovered in $0.2 \mathrm{ml}$ of FACS buffer containing with $0.11 \%$ Trypan Blue and incubated for $1 \mathrm{~min}$ in order to quench the FITC-fluorescent signal 
coming from nanospheres adsorbed to the cell surface. Cells were examined in the flow cytometer and the auto-fluorescence of nontreated cells were used as control.

Cell surface marker evaluation. For the analysis of the surface expression of co-stimulatory molecules, at day 8 of differentiation, BMM- $\varphi$ were incubated with FITC-loaded nanospheres at a concentration of $1 \mathrm{mg} / \mathrm{ml}$ (in polymer). Nonstimulated and lipopolysaccharide $(10 \mu \mathrm{g} / \mathrm{ml})$ treated cells were used as negative and positive controls, respectively. After $20 \mathrm{~h}\left(37^{\circ} \mathrm{C}, 5 \%\right.$ $\mathrm{CO}_{2}$ ), BMM- $\varphi$ were washed thoroughly with cold FACS buffer and then recovered for surface co-stimulatory markers analysis. Antibodies used for assessment of activation included PEconjugated antimouse-CD40, BV-conjugated antimouse-CD86, PerCP/Cy5.5-conjugated antimouse-CD80, Alexa 647-conjugated anti-mouse-MHCII, APC-conjugated antimouseCD206 and PeCy7-conjugated anti-mouse-CD11b antibodies. Dead cells were excluded by staining with 7-A AD and the intensity of the expression of each cell surface marker was reported as mean fluorescence intensity of CD11b+/7-AAD- cells.

Nanospheres internalization pathway. To study the pathways involved in the internalization of nanospheres, BMM- $\varphi$ were preincubated with different inhibitors, listed in Supplementary Table 1 (see online at www.futuremedicine.com/doi/suppl/10.2217/nnm.14.116), for $30 \mathrm{~min}$. After that time, cells were cultured with nonfunctionalized and functionalized $(0.5 \mathrm{mg} / \mathrm{ml})$ PLGA nanospheres. Cells with no inhibitor and with or without nanospheres were used as positive and negative controls, respectively. After $1 \mathrm{~h}$ of incubation, the cells were washed with PBS pH 7.4 and the internalization analyzed by flow cytometry upon 1 min incubation with Trypan Blue. Dead cells were excluded by staining with 7-AAD. The results were reported as mean fluorescence intensity of cells with inhibitor, which were normalized relative to positive control.

Secreted cytokine assay. After $20 \mathrm{~h}$ of stimulation with FITC-loaded non-functionalized or functionalized PLGA nanospheres, cell-free supernatants were collected and assayed for IL-4, IL-6, IL-12p40 and TNF- $\alpha$ by an ELISA assay accordingly to the manufacturer's instructions (Biolegend).

Fluorescence microscopy: Nanospheres internalization pathway. The internalization of FITC-loaded nanospheres was also evaluated by fluorescence microscopy (Leica DMI6000B). $\mathrm{BMM}-\varphi$ were cultured onto 12-mm diameter coverslips on a 24-well plate, and at day 8 of differentiation were treated with nonfunctionalized and functionalized $(0.25 \mathrm{mg} / \mathrm{ml})$ PLGA nanospheres. After specific times, cells were washed with $\mathrm{PBS} \mathrm{pH} 7.4$, to remove noninternalized nanospheres, fixed and then the coverslips were mounted onto slides using DAPI-Vectashield (Vector Laboratories, Inc., CA, USA). The images were overlaid with imaging software ImageJ to visualize co-localization.

Fluorescence microscopy: Nanospheres intracellular trafficking. To study the intracellular trafficking of nanospheres, ВMM- $\varphi$ were cultured, as described previously. After 2 $\mathrm{h}$ the cells were washed and stained with Early Endosomes-RFP or LysoTracker Red for 15 and $30 \mathrm{~min}$, respectively. Cells were then washed with PBS pH 7.4, fixed and the coverslips were mounted onto slides using DAPI-Vectashield (Vector Laboratories, Inc., CA, USA). Cells were observed using a Leica DMI6000B microscope (Leica, Wetzlar, Germany). The images were overlaid with imaging software ImageJ to visualize co-localization.

Parasites \& cell culture. A cloned line of Leishmania infantum (L. infantum, MHOM/MA/67/ITMAP-263) promastigotes, stably expressing the luciferase gene (LUC) and $L$. infantum axenic amastigotes were derived from LUC-expressing promastigotes and were 
grown and used to infect macrophages as already reported [8]. The human leukemia monocyte THP1 cell line was grown and differentiated into macrophages, as described previously [8].

Production of AmB-loaded MN-functionalized PLGA nanospheres. The preparation of AmB-loaded PLGA nanospheres was based on the nanoprecipitation method. Briefly, $25 \mathrm{mg}$ of polymer and $0.5 \mathrm{mg}$ of $\mathrm{AmB}$ (drug loading [w/w] of $2 \%$ ) was dissolved in $4 \mathrm{ml}$ of acetone and $0.2 \mathrm{ml}$ of DMSO, respectively. This organic phase was adjusted to $\mathrm{pH} 4$, using $\mathrm{HCl}$, in order to promote the AmB solubilization and then added to an aqueous solution of $0.5 \%(\mathrm{w} / \mathrm{v})$ of Poly (vinyl alcohol), under magnetic stirring. After solvent evaporation nanospheres were recovered by centrifugation. The functionalization with $\mathrm{MN}$ was achieved by the carbodiimide chemical reaction described above. Nanospheres were sterilized by exposure to UV light during $20 \mathrm{~min}$ and stored at $4^{\circ} \mathrm{C}$ until further use. AmB loading on the functionalized and nonfunctionalized PLGA nanospheres was determined directly by addition of DMSO followed by $A m B$ quantification at $392 \mathrm{~nm}$ by spectrophotometry (Shimadzu $\AA$ UV-2401 PC, EVISA, Duisburg, Germany).

In vitro cytotoxicity on macrophages \& intracellular $L$. infantum amastigotes. The in vitro toxicity of free AmB and respective loaded nanoformulations toward human THP1 differentiated macrophages and murine BMM- $\varphi$ was evaluated using the MTT assay, as previously described [8]. The amount of free $A m B$ was equivalent to the AmB amounts present on the nanospheres. The activity of free and loaded AmB nanoformulations against LUC-expressing amastigotes on human THP1 differentiated macrophages and murine BMM- $\varphi$ was evaluated using a luciferase assay system (Promega, WI, USA) [8].

In vivo studies: Animals, infection \& treatment. Six-week-old male Balb/c mice were purchased from Charles River (Barcelona, Spain). Animals were housed under specific pathogen-free conditions for acclimatization, at the animal resource facilities of IBMC, 1 week before the experiments. For infection of Balb/c mice, $10^{8}$ stationary phase $L$. infantum promastigotes were injected intraperitoneally. Infected mice (6 mice/group) received a singledose treatment of Fungizone ${ }^{\circledR}$ (Life Technologies, ThermoFisher Scientific, Inc., MA, USA) (1 $\mathrm{mg} / \mathrm{kg}$ ) or empty MN-functionalized PLGA nanospheres (1 $\mathrm{mg} / \mathrm{kg}$ ) or AmB-loaded MNfunctionalized PLGA (1 mg/kg of AmB on MN-PLGA nanospheres). After 14 days of infection, mice (3-4 mice/group) were treated by intraperitoneal injection and given the treatment described above. One week after treatment administration, mice were euthanized and spleen and liver were aseptically recovered for parasite quantification by serial dilution assay. The total organ parasite burden was calculated using the weight of the respective organs.

Cytokines production by ELISA \& quantification of total nitrites. The supernatant of 96-h culture of splenocytes $\left(2 \times 10^{5} /\right.$ well) recovered from the differently treated animal groups was used for quantification of cytokines and nitric oxide (NO). Secreted cytokines were quantified in the supernatants after stimulation or not with concanavalin $A$ (ConA; $6 \mu \mathrm{g} / \mathrm{ml}$ ) or soluble Leishmania antigens (SLAs; $25 \mu \mathrm{g} / \mathrm{ml}$ ) by ELISA. IL-10, IFN- $\gamma$, TNF- $\alpha$ and IL-12p40 were measured in all infected untreated and treated mice. Samples were assayed in triplicate, and data were expressed as the mean \pm standard deviation (SD). The NO content was quantified in the supernatants after stimulation with SLAs $(25 \mu \mathrm{g} / \mathrm{ml})$ using the Griess method.

Data analysis. Data were presented as the mean \pm SD and all experiments were independently repeated at least three-times. p-values $<0.05$ (two sided) were considered as statistically significant. For the in vivo studies the statistical significance was assessed using 
one-way ANOVA followed by Dunnett's post-test calculated by GraphPad Prism 6 software (La Jolla, CA, USA).

\section{RESULTS \& DISCUSSION}

Physicochemical characterization of nanospheres. Size and surface charge are the most important characteristics of nanocarriers since they are the major determinants of the cellular uptake mechanism and intracellular fate, defining the nanocarriers in vivo performance [11]. Table 1 summarizes the main physicochemical properties of FITC-loaded nanospheres used in this study. Nonfunctionalized PLGA nanospheres had an average size of $179.9 \pm 5.8 \mathrm{~nm}$, and loading with FITC led to a significant increase $(\mathrm{p}<0.001)$ to $200.1 \pm 6.0 \mathrm{~nm}$. Functionalization of empty PLGA nanospheres with carbohydrates ( $\mathrm{M}$ or $\mathrm{MN}$ ) increase the nanospheres size (194.4 \pm 8.1 [ $p<0.001$ ] $191.2 \pm 2.7$ [p < 0.01], respectively). When FITC was incorporated on the carbohydrate-functionalized PLGA nanospheres, we observed a significant increase ( $p<0.001$ and $p<0.01$ for $M$ and $M N$, respectively) on the average size to values of $211.4 \pm 9.7$ and $202.2<2.4 \mathrm{~nm}$. For the Ms functionalized empty or FITC-loaded PLGA nanospheres the size was identical to nonfunctionalized empty PLGA nanospheres. All prepared nanoformulations exhibited size $(179.9 \pm 5.8$ to $211.4 \pm 9.7 \mathrm{~nm})$ within the range suitable for uptake by macrophages [12]. To evaluate the influence of protein present in cell culture conditions the nanoformulations were incubated for $24 \mathrm{~h}$ at $37^{\circ} \mathrm{C}$ in the presence of $10 \%$ FBS and no statistically significant changes were observed when compared with the formulations at buffered conditions.

The polydispersity index (PDI) characterizes the nanocarriers in terms of size homogeneity by a value of the relative variance of sizes within the population. The nonfunctionalized and functionalized PLGA nanospheres obtained were mono-disperse, with PDI values always below 0.1 (Table 1), suggesting a relatively uniform size.

The nonfunctionalized nanospheres exhibited a $\zeta$-potential of $-16.5 \pm 0.5 \mathrm{mV}$, which was neither affected with the incorporation of FITC, nor with the carbohydrate functionalization $(\mathrm{p}=$ $0.1154)$, consistent with the presence of carboxyl groups that remained free. Under normal $\mathrm{pH}$ conditions FITC confers partial negative charges. Indeed, the incorporation of FITC on the carbohydrate-functionalized nanospheres lead to a modest $(p<0.01)$ increase on the net negative surface charge of the nanocarriers, except for $\mathrm{MN}$-functionalization. In this case, no differences were found on the surface charge of the nanocarriers, which could be explained by masking of the negatively charged carboxyl end groups following esterification with MN [9]. The surface charge can influence particle stability, and is widely described that high values, either positive or negative, tend to stabilize the nanocarriers to a greater extent, as is the case of our functionalized FITC-loaded PLGA nanospheres ( -2 $4 \mathrm{mV}$ ).

The carbohydrates moieties functionalized nanospheres were confirmed using Fourier transform infrared spectroscopy, to quantitatively determine successful linker and carbohydrate conjugation to the nanospheres surface. The Fourier transform infrared spectra obtained for carbohydrate functionalized PLGA nanospheres present a prominent peak at $1630 \mathrm{~cm}^{-1}$, representing the covalent binding that is not observed in the spectrum of nonfunctionalized nanospheres (Supplementary Figure 1). The total amount of carbohydrate attached to the nanospheres surface was quantified by phenol-sulfuric acid ( $M$ and $M N$ ) or the ninhydrin assay (Ms) and was normalized to the total mass of nanospheres. The amount of carbohydrate conjugated to the PLGA nanospheres were $761.4 \pm 13.1,929.1 \pm 34.8$ and 
$822.0 \pm 18.5 \mu \mathrm{g}$ of $\mathrm{M}, \mathrm{MN}$ and $\mathrm{Ms}$, respectively, per $\mathrm{mg}$ of nanospheres. When PLGA nanospheres had FITC incorporated, a decrease in the amount of carbohydrate was found to levels of $546.4 \pm 7.3,761.7 \pm 43.7$ and $743.7 \pm 22.6 \mu \mathrm{g}$ of $\mathrm{M}, \mathrm{MN}$ and Ms, respectively, per milligram of nanospheres. The carbohydrate functionalization is conducted under acidic conditions that may contribute to FITC protonation, weakening the carbohydrate attachment to the nanospheres surface.

The morphological analysis by TEM confirmed the presence of spherical particles with a size similar to that obtained by dynamic light scattering (Figure 1A-D \& Table 1). To study the presence and orientation of carbohydrates in the surface of PLGA nanospheres (Figure 1E). FITC loading was calculated to be $217.9 \pm 20.7 \mu \mathrm{g}$ FITC per $\mathrm{mg}$ of nanospheres with an encapsulation efficiency close to $56 \%$. The integrity and in vitro release of FITC after its incorporation in the PLGA nanospheres was evaluated, as FITC is not covalently bound to the polymer. The fluorescence spectrum was similar to free FITC (data not shown) and the in vitro release assay revealed that only around 3\% of total incorporated FITC is released during 6 days at $37^{\circ} \mathrm{C}$ (Figure $\left.1 \mathrm{~F}\right)$.

The effect of surface functionalization on the uptake of PLGA nanospheres by BMM- $\varphi$. The expression of MR on the primary culture of $B M M-\varphi$ was studied by flow cytometry. The data revealed that MR expression is time-dependent (Figure 2A). At day 8 of BMM- $\varphi$ differentiation was observed the maximum expression of MR $(77.9 \%$ of the whole cultured cells) in the $\mathrm{F} 4 / 80+$ population. In the following studies BMM- $\varphi$ with 8 days of differentiation was used.

The cellular uptake of nonfunctionalized and functionalized PLGA nanospheres was followed by flow cytometry. According to the in vitro release data (Figure $1 \mathrm{~F})$ minimum release $(<5 \%)$ of FITC occurs, indicating that for all fluorescence in PLGA nanospheres observed is due to their internalization. The internalization of the nonfunctionalized and functionalized PLGA nanospheres was time dependent (Figure 2B). As early as 30-min postincubation, nanospheres were found within the cells CD11b+/FITC+ and it appears that a saturation limit was reached for nonfunctionalized nanospheres at about $2 \mathrm{~h}$ (Figure $2 \mathrm{~B} \& \mathrm{C}$ ). Evidence of saturation of nanocarriers uptake has been previously documented in the literature for other cell types [13]. The PLGA nanospheres surface functionalization led to an increase $(M, p<$ $0.01)$; $M N$ and $M s(p<0.001)$ in the level of uptake of the nanospheres by the BMM- $\varphi$ when compared with the nonfunctionalized nanospheres, after $24 \mathrm{~h}$. There are no significant differences in the uptake between the three carbohydrate-functionalized PLGA nanospheres. In delivery systems, cellular uptake profile will reflect the delivery efficiency and the bioavailability of the nanocarriers.

The fluorescence microscopy images confirmed the internalization of the nonfunctionalized and functionalized PLGA nanospheres by BMM- $\varphi$ after $24 \mathrm{~h}$ of incubation, as shown by the presence of green dots within the cytoplasm (Figure $2 \mathrm{C}-\mathrm{E}$ ).

Lysosomal fate of the PLGA nanospheres on BMM- $\varphi$. In general, the intracellular outcome of nanocarriers is strongly affected by the route of entry in the cell. Remarkably, depending on the physicochemical characteristics, the internalization pathway as well as the intracellular fate of nanocarriers, is a key issue for the drug to be efficiently delivered. The release of the drug into the enzymatic environment of the lysosomes or directly in the cell cytoplasm will, indeed, have impact on the pharmacological activity. To determine whether the uptake mechanism of PLGA nanospheres entry, into 8-day differentiated BMM- $\varphi$ was mediated by endocytosis, the cells were incubated with nonfunctionalized and functionalized PLGA 
nanospheres and maintained at $4^{\circ} \mathrm{C}$, since endocytosis as an energy dependent process, is blocked at low temperatures. We observed that the uptake of all PLGA nanospheres was significantly decreased at $4^{\circ} \mathrm{C}$ (Figure $3 \mathrm{~A}$ ), suggesting that their uptake is mediated by endocytosis. Several endocytic pathways for macromolecules are identified so far: clathrinmediated endocytosis, caveolae-mediated endocytosis, macropinocytosis and clathrin- and caveolae-independent endocytosis [11]. In order to identify the uptake mechanisms involved in the cellular entry of functionalized PLGA nanospheres, we employed several specific endocytic inhibitors (Supplementary Table 1). When BMM- $\varphi$ were preincubated with chlorpromazine, an inhibitor of clathrin-mediated endocytosis, and then with nonfunctionalized and functionalized PLGA nanospheres, the uptake was reduced by $20-40 \%$, respectively, compared with nontreated cells (Figure 3A). Among the different carbohydrates, the inhibition was more pronounced with MN-functionalized nanospheres ( 40\%). Similarly, when cells were pretreated with hypertonic conditions $(0.45 \mathrm{M}$ sucrose), also an inhibitor of clathrinmediated endocytosis, uptake of all PLGA nanospheres by the BMM- $\varphi$ was reduced by approximately $40 \%$, in relation to the nontreated cells. Sucrose leads to a more pronounced effect on the uptake of nonfunctionalized nanospheres, which suggests that the mechanism of action the chlatrin-mediated endocytosis inhibitors influence the overall effect of the internalization. Indeed, it has been described that hyperosmotic sucrose acts by preventing clathrin and adaptors from interacting; while cationic amphiphilic drugs as chlorpromazine cause a decrease in coated pits on the cell surface and clathrin lattices assembly on endosomal membranes. In our work, ВMM- $\varphi$ treatment with hyperosmotic sucrose leads to approximately $40 \%$ inhibition of nonfunctionalized PLGA nanosphere internalization (Figure $3 \mathrm{~A}, 0.62 \pm 0.11$ ), indicating that the few coated pits remaining functional after hyperosmotic sucrose are sufficient to internalize $60 \%$ of the nanospheres. Chlorpromazine treatment of BMM resulted in approximately $20 \%$ inhibition of nonfunctionalized PLGA nanosphere internalization (Figure $3 \mathrm{~A}, 0.81 \pm 0.05$ ). The preincubation of the cells with filipin III, a selective inhibitor of caveolae formation, only influenced the uptake of MN-functionalized PLGA nanospheres by the BMM- $\varphi$ with approximately $20 \%$ inhibition, when compared with nontreated cells (Figure 3B). When cells were preincubated with cytochalasin D, a macropinocytosis inhibitor, and then with each type of PLGA nanospheres under study, no inhibitory effect was observed in the uptake, as compared with the nontreated cells (Figure 3B). Taken together, these studies suggest that the internalization of nonfunctionalized and functionalized PLGA nanospheres occurs, mainly, through clathrin-mediated endocytosis. In particular, MN-functionalization lead to more than one cellular uptake mechanism, clathrinand caveolae-mediated endocytosis are likely to take place in the translocation processes of these nanoparticles across the lipid bilayer of the cell plasma membranes. This outcome can partly be explained by nanospheres size, which is a major parameter defining nanocarriers endocytosis process and is well known that the diameter of clathrin-coated vesicles ranges between 100 and $200 \mathrm{~nm}$ while caveosomes have a general diameter of approximately 60$100 \mathrm{~nm}$ [11]. It should be noticed that the discrepancy between the typical endosome sizes described in literature and the actual size of nanocarriers can be explained on fundamental biological processes, involving pinocytosis, thus imposing less mechanical constraints on the vesicle formation.

Certain internalization pathways, such as clathrin-mediated endocytosis, may lead to a complete degradation of the nanocarriers at the later lysosomal stage. To identify the intracellular trafficking of the nonfunctionalized and functionalized PLGA nanospheres, we performed co-localization assays with EEA1 and LysoTracker, as known markers for early endosomes and lysosomes, respectively. As shown in Figure 3D-G, co-localization (yellow color) of FITC-loaded PLGA nanospheres with LysoTracker-red demonstrated that the particles are primarily transported to lysosomes following endocytosis by BMM- $\varphi$. Few co- 
localization signals were observed in EEA1 (data not shown), which indicates that all types of PLGA nanospheres are not typically localized within early endosomes. Lysosomotropic agents were used to clarify the involvement of the endosomal/lysosomal compartments in the internalization of the nanospheres by BMM- $\varphi$. When BMM- $\varphi$ were pretreated with ammonium chloride and then incubated with nonfunctionalized and functionalized FITC-loaded PLGA nanospheres a significant inhibition in the uptake was observed (Figure 3C). The carbohydrate functionalization of the PLGA nanospheres, in particularly, MN- and Ms-, is more dependent of the lysosome compartment when compared with nonfunctionalized nanospheres. Taken together these data, it is proposed that all the PLGA nanospheres used in this study undergo endosomal internalization, trafficking to the lysosome. Degradation of nanocarries in lysosomes plays an important role in drug delivery to intracellular pathogens [14]. Within lysosomes the nanocarriers are degraded into smaller fragments and the incorporated drug/agent can be released through surface or bulk erosion of the degradable polymers, promoting a sustained drug release from the lysosomal compartments, which could be highly advantageous for drug delivery applications. Particle surface charge determines the nanocarriers trafficking in cells and it was described that anionic but not cationic nanocarriers transited through the lysosomal pathway [15], which is consistent with our findings.

MN-functionalized PLGA nanospheres enhanced BMM- $\varphi$ expression of MHC II, \& costimulatory molecules. Contrary to DCs, macrophages do not have a constitutive expression of migratory and co-stimulatory molecules, their expression at macrophages surface occurs only upon activation [16]. Thus, we assess by flow cytometry the expression of these specific surface markers to determine the influence of nonfunctionalized and functionalized PLGA nanospheres in the activation status of $B M M-\varphi$. Here, we observe increased expression of surface markers associated with antigen processing and presentation (MHCII) and T-cell costimulation (CD86, CD80 and CD40) in BMM- $\varphi$ upon $20 \mathrm{~h}$ of incubation with functionalized PLGA nanospheres, as compared with nonfunctionalized nanospheres (Figure 4A-D). When BMM- $\varphi$ were cultured with functionalized PLGA nanospheres we also observed an increase on the surface expression of the MR in comparison to nonfunctionalized nanospheres (Figure $4 \mathrm{~F}$ ). This evidence indicates a positive self-regulation of the CLR, upon MR recognition, by the carbohydrate, which is supported by the fact that CLRs undergo internalization and recycling between the plasma membrane and the endosomal compartments [5]. Another interesting observation is that the MN-functionalization of PLGA nanospheres induced higher expression of $\mathrm{MHCll}$ and co-stimulatory molecules as compared with M-functionalized nanospheres. Previously, it has been reported that soluble carbohydrate and nonfunctionalized nanospheres plus soluble carbohydrates did not change cell surface marker expression, endorsing the contribution of the covalent bond of the carbohydrate to the nanospheres surface [15]. Several other types of carbohydrates (lactose, galactose) have been used to functionalize the surface of nanocarriers targeting MR on macrophages, with concomitant cell activation [5].

Although the expression of MHCII, CD40, CD80 and CD86 in macrophages is essential, it is not sufficient to induce T-cell activation. Thus, it is crucial that the activation of macrophages leads to the production of different cytokines, in order to favor the development of a more effective immune response. In particular, the production of pro-inflammatory cytokines is essential for the activation of macrophages, to stimulate their antimicrobial properties and to initiate adaptive immune responses. The incubation of 8-day differentiated BMM- $\varphi$ for $20 \mathrm{~h}$ with either nonfunctionalized or functionalized PLGA nanospheres loaded with FITC increased the secretion of pro-inflammatory cytokines IL-6, IL12p40 and TNF- $\alpha$, in comparison to the nonstimulated controls (Figure 4F-H). The greatest amounts of IL-6 and TNF- $\alpha$ were produced by BMM- $\varphi$ stimulated with MN-functionalized PLGA nanospheres (Figure 4F \& G). These results are in agreement with studies demonstrating that the targeting of $M R$ in 
macrophages resulted in the production of pro-inflammatory cytokines [5]. Interestingly, the functionalization with the different carbohydrates increased the secretion of IL-4, a Th2 cytokine, by BMM- $\varphi$ when compared with nonfunctionalized nanospheres (Figure 4I). Levels of IFN- $y$ for M, MN and Ms-PLGA nanospheres were $1760 \pm 72,2100 \pm 54$ and $2240 \pm 41$ $\mathrm{pg} / \mathrm{ml}$, respectively, which supports the immune-modulatory role of the nanospheres toward a Th1 response. The presence of IL-4 in extravascular tissues promotes MR expression on macrophages [17]. In our results, the carbohydrate functionalization leads to an increase of MR expression on BMM- $\varphi$ which can be associated to the secretion of IL- 4 and the continuous uptake of the functionalized PLGA nanospheres through clathrin-mediated endocytosis. Together, these observations indicate that carbohydrate functionalization of PLGA nanospheres enhanced BMM- $\varphi$ activation. In particular, MN- or Ms-functionalization was sufficient to activate $B M M-\varphi$, suggesting that surface charge contributes to the activation. Indeed, carbohydrate functionalization of polyanhydride nanoparticles was described to enhance alveolar macrophage activation as compared with nonfunctionalized particles, accordingly to the nanoparticle surface charge [5].

To this point, it was demonstrated that carbohydrate functionalization of PLGA nanospheres, particularly with $\mathrm{MN}$, improve their immunotherapeutic effect on BMM- $\varphi$, through the induction of a more efficient activation of macrophages, which could lead to the development of a robust immune response. These observations lead us to pursue a biological application that could benefit from immune-modulatory properties of the MN-functionalized PLGA nanospheres. The intracellular parasite Leishmania spp. has the ability to survive and multiply within DCs and macrophages, and for that is consider an ideal model to study obligate intracellular microbes (Mycobacterium tuberculosis, Listeria monocytogenes, Salmonella typhimurium, Toxoplasma gondii, Trypanosoma cruzi and HIV). An immune-modulatory nanocarrier, such as $\mathrm{MN}$ functionalized PLGA nanospheres, that target APCs, can be an attractive approach for the direct targeting of chemotherapeutics to VL infected cells.

Production of AmB-loaded MN-functionalized PLGA nanospheres. Previous reports from our laboratory have described the ability of nonfunctionalized PLGA nanospheres to improve in vivo efficacy of AmB on a VL experimental model [10]. In the present work, we sought to extend our findings by asking if functionalization with specific carbohydrates (MN was the selected one) provides additional immune-stimulatory ability to the PLGA nanospheres and thus improving AmB efficacy.

We have employed the nanoprecipitation technique to incorporate AmB on $\mathrm{MN}$-functionalized PLGA nanospheres. The physicochemical properties of the nanospheres were studied ( Table 2 ). The incorporation of AmB did not affect the physicochemical properties of the MNfunctionalized PLGA nanospheres and was possible to achieve about $57.5 \pm 4.3 \%$ encapsulation efficiency with an initial drug loading of $2 \%$, corresponding to $5.8 \pm 0.4 \mu \mathrm{g} \mathrm{AmB}$ per mg of PLGA. Others have reported encapsulation values above 50\% for AmB in PLGA nanospheres with higher initial drug loading values (10-30\%) [18]. AmB is a highly hydrophobic drug contributing to the successful application of the nanoprecipitation technique, as indicated by the encapsulation efficiency value. We can also consider that the possible electrostatic interaction between capped carboxyl groups of PLGA and the positively charged primary amine group of $\mathrm{AmB}$ may contribute to the incorporation of $\mathrm{AmB}$ on the nanospheres. No morphological differences were observed by TEM with the incorporation of AmB on the MN-functionalized PLGA nanospheres (Supplementary Figure 2).

In vitro \& in vivo efficacy of AmB-loaded MN-functionalized PLGA nanospheres. The in vitro cytotoxicity of AmB (free form and loaded in MN-PLGA nanospheres) was evaluated in 
human THP1 differentiated macrophages and murine BMM- $\varphi$ (Supplementary Figure 3). Free AmB is able to inhibit $50 \%$ of the cell growth at $19.8 \pm 3.6$ and $21.8 \pm 2.8 \mu \mathrm{g} / \mathrm{ml}$ for THP1 and BMM- $\varphi$, respectively. When loaded on MN-functionalized PLGA nanospheres, a significant reduction of AmB toxicity was observed, both on human and murine macrophages with IC50 values higher than $50 \mu \mathrm{g} / \mathrm{ml}$. In the mammalian host, the parasite $L$. infantum resides inside a phagolysosome. Both free $\mathrm{AmB}$ and its nanoformulation demonstrated to be efficient in reducing the intracellular growth of $L$. infantum amastigotes on THP1 and BMM- $\varphi$ (Table 3 ). Moreover the AmB-loaded MN-functionalized PLGA nanospheres were able to retain free AmB efficacy and to improve it by 70 - and 94-fold in intracellular L. infantum amastigotes in THP1 differentiated macrophages and $B M M-\varphi$, respectively. No toxicity was observed $72 \mathrm{~h}$ after treatment with up to $2 \mathrm{mg} / \mathrm{ml}$ in polymer toward the mammalian cells and intracellular $L$. infantum amastigotes. Together these observations indicate that the incorporation of AmB on $\mathrm{MN}$-functionalized PLGA nanospheres reduced its drug cellular toxicity on macrophages and retained and improved its antiparasitic activity, which, and most probably, allow application of higher doses, improving AmB efficacy and safety.

Next, we assessed the efficacy of AmB-loaded MN-functionalized PLGA nanospheres in a VL susceptible murine model. After acute infection (14 days after the infection), single dose at 1 $\mathrm{mg} / \mathrm{kg}$ in AmB was administered via intraperitoneal (ip.) injection, draining the nanocarriers or larger compounds (MW >20 kDa) through the lymphatic ducts. Here, we thus expected that the nanocarriers reach the systemic circulation via the lymphatics relatively quickly, due to their small size. One week upon the administration, we have observed that the group receiving AmB-loaded MN-functionalized PLGA nanospheres had significant reduction ( $\mathrm{p}<$ 0.001 ) on the parasite load on both splenic and liver tissues (Figure 5A \& B), when compared with intraperitoneal AmB (Fungizone) treated and control, empty MN-functionalized PLGA nanospheres groups. Indeed, a single dose of AmB-loaded MN-functionalized PLGA nanospheres promoted a reduction of $99.1 \pm 1.3 \%$ and $99.5 \pm 1.1 \%$, in the spleen and liver, respectively; as compared with the vehicle control. Specifically, in the spleen the untreated group had a log of $8.86 \pm 0.45$ parasites $/ g$ while the functionalized-treated group had $5.37 \pm$ 0.20 parasites/g; the data were similar in the liver: $8.43 \pm 0.32$ and $4.78 \pm 0.15$ parasites $/ \mathrm{g}$, respectively. Nonfunctionalized PLGA nanospheres loaded with AmB were also able to lower both splenic and hepatic parasites by 2.5- and 2-logs, respectively (unpublished results, Supplementary Figure 4). The new AmB nanoformulations has demonstrated efficacy in shortterm treatment without achieving cure. A different treatment scheme associated with a longer term treatment could lead to control of the infection and eventually cure. It is relevant to note that the MN-functionalized PLGA nanospheres loaded with AmB combining AmB therapeutic efficacy with macrophage activation was more efficient than Fungizone, currently used in the VL treatment.

As IFN-y and IL-10 are described to be essential cytokines governing the fate of the infection in $\mathrm{VL}$, we quantified their secretion by splenocytes recovered from all treatment groups. Indeed, AmB-loaded MN functionalized PLGA nanospheres treated mice exhibited IFN-Y levels significantly higher, in comparison with Fungizone or empty MN-functionalized PLGA nanospheres treated infected mice (Figure $5 \mathrm{C}$ ). The IFN- $\mathrm{F} / \mathrm{IL}-10$ ratio was higher in functionalized-nanoparticles treated mice than in untreated, AmB- or empty nanospheres treated animals. To unravel the status of the IFN- $/ / \mathrm{IL}-10$ ratio in functionalized-nanoparticles treated mice, levels of TNF- $\alpha$ and nitric oxide were also quantified. The AmB-loaded MNfunctionalized PLGA nanospheres treated mice had a 2.3-fold increase in TNF- $\alpha$ in comparison with the infected untreated mice, while Fungizone treated mice showed a 1.8-fold, when compared with infected untreated mice (Figure $5 \mathrm{E}$ ). This results in an increase of NO production (Figure 5F). The AmB-loaded MN-functionalized PLGA nanospheres treated mice 
had a fourfold increase in NO production, in comparison with the infected untreated mice, while Fungizone treated mice showed a threefold increase, when compared with infected untreated mice (Figure 5E). In VL, macrophages are both the host cell for Leishmania replication and the effector cell for parasite killing. When macrophages become infected are unable to trigger a pro-inflammatory response, but the delivery of AmB to these macrophages will enable parasite killing by apoptosis. To be successful, this process also needs to be supplemented with TNF-a, iNOS should be induced, IL10 has to be neutralized leading to the production of IFN-Y [19]. Indeed, our results show that AmB-loaded MN-functionalized PLGA nanospheres treatment of experimental VL led to the rapid killing of parasites in macrophages, and to increased IFN- $\gamma$, TNF- $\alpha$ and NO levels.

Shimizu et al. have demonstrated that the i.p. administration of oligomannose-coated liposome-entrapped SLAs strongly induces an antigen-specific Th1 immune response in BALB/c mice [20]. In our study, the MN-functionalization of the PLGA nanospheres increased MHCIl expression triggering a Th1-like pathogen specific immune response, and together with the AmB loading, these nanocarriers not only reduced, in vitro, the effective drug dose and toxicity, it also activated the macrophages to commit to a Th1 response leading to an effective adaptive immune response against this intracellular pathogen.

\section{CONCLUSION}

In conclusion, we demonstrated that carbohydrate functionalization of PLGA nanospheres leads to immune-modulatory properties on macrophages, eliciting a pro-inflammatory response, upon clathrin-mediated endocytosis. Efficient uptake by macrophages led to lysosome accumulation that acts as a perfect reservoir for continuous exposure of an intracellular pathogen to a delivered drug. As a proof of concept, the AmB-loaded on MNfunctionalized PLGA nanospheres confirmed superior in vivo efficacy, in comparison with Fungizone, against VL infection. A single injection and a 1-week course of treatment were sufficient to achieve significant decrease on the visceral organs parasite load. The pharmacologically activity of AmB was improved by MN-functionalized PLGA nanospheres, that triggered host immune responses to parasite killing resulting in a promising therapy for VL. The developed MN-functionalized nanocarriers could represent a huge advantage in the treatment strategy of obligate intracellular pathogens, which continue to have a major negative impact on public health.

\section{FUTURE PERSPECTIVE}

Targeting CLRs by surface functionalization of polymeric nanospheres represents a promising approach to modulate the immune system. Carbohydrates commonly found on the surface of pathogens, such as $\mathrm{MN}$, can significantly enhance the interaction of polymeric nanospheres and macrophages by further upregulating surface expression markers, T-cell co-stimulatory molecules and the secretion of proinflammatory cytokines. For intracellular pathogens, delivery of an active agent associated with a controlled proinflammatory immune response may be highly beneficial. With the inherent advantage described in this work of enhanced therapeutic efficacy, the proposed $\mathrm{MN}$-functionalized polymeric nanospheres represent an interesting nanoplatform for intracellular pathogens. Nevertheless, deeper understanding on how these carbohydrate-functionalized nanospheres interact with the immune cells will be highly valuable. 


\section{FINANTIAL \& COMPETING INTERESTS DISCLOSURE}

This work was supported by Fundo Europeu de Desenvolvimento Regional (FEDER) funds through the Operational Competitiveness Program-COMPETE and by national funds through Fundação para a Ciência e a Tecnologia under projects FCOMP-01-0124-FEDER-015718 (PTDC/SAU-ENB/113151/2009). D Barros was supported by FCOMP-01-0124-FEDER015718 (PTDC/SAU-ENB/113151/2009) project. SA Costa Lima was supported by SFRH/BPD/37880/2007 and by QREN under contract NORTE-07-0124-FEDER-000067. The authors have no other relevant affiliations or financial involvement with any organization or entity with a financial interest in or financial conflict with the subject matter or materials discussed in the manuscript apart from those disclosed.

No writing assistance was utilized in the production of this manuscript.

\section{ETHICAL CONDUCT OF RESEARCH}

The authors state that they have obtained appropriate institutional review board approval or have followed the principles outlined in the Declaration of Helsinki for all human or animal experimental investigations. In addition, for investigations involving human subjects, informed consent has been obtained from the participants involved.

\section{REFERENCES}

Papers of special note have been highlighted as: • of interest; •• of considerable interest

1. Mahapatro A, Singh DK. Biodegradable nanoparticles are excellent vehicle for site directed in vivo delivery of drugs and vaccines. J. Nanobiotechnology 28, 55-66 (2011).

2. Danhier F, Ansorena E, Silva JM et al. PLGA-based nanoparticles: an overview of biomedical applications. J. Control. Release 161, 505-522 (2012).

3. Foged C, Hansen J, Agger EM. License to kill: formulation requirements for optimal priming of CD8(+) CTL responses with particulate vaccine delivery systems. Eur. J. Pharm. Sci. 45, 482-491 (2012).

4. Yang Y W, Hsu PY. The effect of poly(d,I-lactide-co-glycolide) microparticles with polyelectrolyte self-assembled multilayer surfaces on the cross-presentation of exogenous antigens. Biomaterials 29, 2516-2526 (2008).

5. Chavez-Santoscoy A, Roychoudhury R, Pohl $\mathrm{N}$ et al. Tailoring the immune response by targeting C-type lectin receptors on alveolar macrophages using "pathogen-like" amphiphilic polyanhydride nanoparticles. Biomaterials 33, 4762- 4772 (2012). - Representative of carbohydrate-functionalized delivery systems based on polymeric nanoparticles as immune-modulatory agents. 
6. Moretton MA, Chiappetta DA, Andrade F et al. Hydrolyzed galactomannan-modified nanoparticles and flower-like polymeric micelles for the active targeting of rifampicin to macrophages. J. Biomed. Nanotechnol. 9, 1076 -1087 (2013). • Representative of carbohydrate-functionalized delivery systems based on polymeric nanoparticles as immune-modulatory agents.

7. Xiao $B$, Laroui $H$, Ayyadurai $S$ et al. Mannosylated bioreducible nanoparticle-mediated macrophage-specific TNF- $\alpha$ RNA interference for IBD therapy. Biomaterials 34, 7471-7482 (2013). - Representative of carbohydrate-functionalized delivery systems based on polymeric nanoparticles as immune-modulatory agents.

8. Costa Lima SA, Resende M, Silvestre $R$ et al. Characterization and evaluation of BNIPDaoct-loaded PLGA nanoparticles for visceral leishmaniasis: in vitro and in vivo studies. Nanomedicine (Lond.) 7, 1839 -1849 (2012).

9. Ghotbi Z, Haddadi A, Hamdy S et al. Active targeting of dendritic cells with mannandecorated PLGA nanoparticles. J. Drug Target 19, 281-292 (2011).

10. Costa Lima SA, Silvestre R, Barros D et al. Crucial CD8+ T-lymphocyte cytotoxic role in amphotericin B nanospheres efficacy against experimental visceral leishmaniasis. Nanomedicine 10 (5), 1021-1030 (2014). • Describes the in vivo efficacy of AmB-loaded nonfunctionalized poly(d,I-lactic-co-glycolic acid) nanospheres on a visceral leishmaniasis experimental model.

11. Hillaireau H, Couvreur P. Nanocarriers' entry into the cell: relevance to drug delivery. Cell Mol. Life Sci. 66, 2873-2896 (2009). • Representative of cell nanoparticles' mechanism of entry and intracellular pathway.

12. Yue $H$, Wei $W$, Yue $Z$ et al. Particle size affects the cellular response in macrophages. Eur. J. Pharm. Sci. 41, $650-657$ (2010).

13. Cartiera MS, Johnson KM, Rajendran V et al. The uptake and intracellular fate of PLGA nanoparticles in epithelial cells. Biomaterials 30, 2790-2798 (2009). • Representative of cell nanoparticles' mechanism of entry and intracellular pathway.

14. Petros R A, DeSimone JM. Strategies in the design of nanoparticles for therapeutic applications. Nat. Rev. Drug Discov. 9, 615-627 (2010).

15. Yue ZG, Wei W, Lv PP et al. Surface charge affects cellular uptake and intracellular trafficking of chitosan-based nanoparticles. Biomacromolecules 12, 2440-2446 (2011). - Representative of cell nanoparticles' mechanism of entry and intracellular pathway.

16. Hamdy S, Haddadi A, Hung RW et al. Targeting dendritic cells with nano-particulate PLGA cancer vaccine formulations. Adv. Drug Deliv. Rev. 63, 943-955 (2011).

17. Adams EW, Ratner DM, Seeberger PH et al. Carbohydrate-mediated targeting of antigen to dendritic cells leads to enhanced presentation of antigen to T cells. Chem. Bio. Chem. 9, 294-303 (2008). 
18. Van de Ven $\mathrm{H}$, Paulussen $\mathrm{C}$, Feijens $\mathrm{P}$ et al. PLGA nanoparticles and nanosuspensions with amphotericin B: potent in vitro and in vivo alternatives to Fungizone and AmBisome. J. Control. Release 161, 795-803 (2012).

19. Sacks D, Noben-Trauth $N$. The immunology of susceptibility and resistance to L. major in mice. Nat. Rev. Immunol. 2, 845 - 858 (2002).

20. Shimizu Y, Takagi H, Nakayama T et al. Intraperitoneal immunization with oligomannosecoated liposome-entrapped soluble leishmanial antigen induces antigen-specific T-helper type immune response in BALB/c mice through uptake by peritoneal macrophages. Parasite Immunol. 29, 229-239 (2007). •• Reports that carbohydrate-functionalized nanocarriers stimulate Th1-like pathogen-specific immune response resulting in an effective adaptive immune response against Leishmania intracellular parasite.

\section{TABLES \& FIGURES}

Table 1. Physicochemical properties of nonfunctionalized and functionalized poly(d,I-lactide-co-glycolide) nanospheres empty or loaded with FITC.

\begin{tabular}{|c|c|c|c|c|c|}
\hline Nanospheres & Size $(n m)^{\dagger}$ & $\mathrm{PDI}^{+}$ & $\begin{array}{l}\zeta \text {-potential } \\
(\mathrm{mV})^{\dagger}\end{array}$ & $\begin{array}{l}\text { Carbohydrate }(\mu \mathrm{g} / \\
\mathrm{mg})^{\dagger}\end{array}$ & $\mathrm{EE}(\%)^{t, t}$ \\
\hline PLGA & $179.9 \pm 5.8$ & $0.052 \pm 0.003$ & $-16.5 \pm 0.5$ & NA & NA \\
\hline PLGA FITC & $200.1 \pm 6.0 * * *, 5$ & $0.069 \pm 0.006$ & $-17.7 \pm 1.2$ & NA & $56.4 \pm 4.1$ \\
\hline M-PLGA & $194.4 \pm 8.1^{* * *, \pi}$ & $0.047 \pm 0.002$ & $-18.2 \pm 0.8$ & $761.4 \pm 13.1^{*, * \star * *, 5}$ & NA \\
\hline M-PLGA FITC & $211.4 \pm 9.7^{* \star *, 5}$ & $0.070 \pm 0.010$ & $-23.2 \pm 1.5^{* * *, 5}$ & $546.4 \pm 7.3^{\#}$ & $56.1 \pm 4.2$ \\
\hline MN-PLGA & $191.2 \pm 2.7 * *, n$ & $0.081 \pm 0.005$ & $-21.05 \pm 4.0$ & $929.1 \pm 34.8^{*, * *, 5}$ & NA \\
\hline MN-PLGA FITC & $202.2 \pm 3.1^{* *, 5}$ & $0.073 \pm 0.003$ & $-24.8 \pm 1.1$ & $761.7 \pm 43.7^{*}$ & $56.0 \pm 4.2$ \\
\hline Ms-PLGA & $182.3 \pm 5.3$ & $0.082 \pm 0.002$ & $-19.8 \pm 1.2$ & $822.0 \pm 18.5^{\ddagger 1, *}$ & NA \\
\hline Ms-PLGA FITC & $192.0 \pm 5.6$ & $0.095 \pm 0.005$ & $-24.1 \pm 2.3^{* *, 5}$ & $743.7 \pm 22.6^{\ddagger \pm}$ & $56.1 \pm 4.1$ \\
\hline
\end{tabular}

tValues represent mean $\pm \mathrm{SD}(\mathrm{n}=3)$.

"Indirect quantification by fluorimetry.

statistically significant when compared with empty nanospheres.

"Statistically significant when compared with empty nonfunctionalized nanospheres.

"Indirect quantification by phenol-sulfuric acid reaction.

*Indirect quantification by ninhydrin assay.

${ }^{*} \mathrm{p}<0.05 ;{ }^{* *} \mathrm{p}<0.01 ;{ }^{* * *} \mathrm{p}<0.001$, statistically significant.

EE: Encapsulation efficiency; FITC: Fluorescein isothiocyanate isomer l; M: Mannose; MN: Mannan; Ms: Mannosamine; NA: Not applicable; PDI: Polydispersity index; PLGA: Poly(D,-lactide-co-glycolide); SD: Standard deviation. 
Table 2. Physicochemical properties of empty and amphotericin B-loaded mannan-functionalized poly(d,I-lactide-co-glycolide) nanospheres.

\begin{tabular}{llllll} 
Nanospheres & Size $(\mathrm{nm})^{\dagger}$ & PDI $^{\dagger}$ & $\zeta$-potential $(\mathrm{mV})^{\dagger}$ & Carbohydrate $(\mu \mathrm{g} / \mathrm{mg})^{\mathrm{t}, \boldsymbol{E}}$ & $\mathrm{EE}(\%)^{\mathrm{t}, \mathrm{s}}$ \\
\hline MN-PLGA & $191.2 \pm 2.7$ & $0.081 \pm 0.005$ & $-21.05 \pm 4.0$ & $929.1 \pm 34.8$ & - \\
\hline MN-PLGA AmB & $180.1 \pm 0.1$ & $0.086 \pm 0.006$ & $-20.8 \pm 1.3$ & $922.9 \pm 2.4$ & $57.5 \pm 4.3$ \\
\hline
\end{tabular}

"Values represent mean \pm SD $(n=3)$

"Indirect quantification by phenol-sulfuric acid reaction.

'Direct quantification by spectrophotometry.

AmB: Amphotericin B; MN: Mannan; PLGA: Poly(D,L-lactide-co-glycolide); PDI: Polydispersity index.

Table 3. Biological effect of amphotericin B in free drug form and loaded on mannan-functionalized poly(d,I-lactide-co-glycolide) nanospheres on Leishmania infantum intracellular amastigotes.

\begin{tabular}{lll} 
Formulation & \multicolumn{2}{c}{$\mathrm{IC}_{50}(\mathrm{nM})$} \\
\cline { 2 - 3 } & $\mathrm{THP1}$ & $\mathrm{BMM}-\varphi$ \\
\hline Free AmB & $1321.08 \pm 151.60$ & $1223.62 \pm 97.46$ \\
\hline MN-PLGA & $>10,000$ & $>10,000$ \\
\hline MN-PLGA AmB & $19.28 \pm 5.51^{*}$ & $13.10 \pm 0.87^{*}$
\end{tabular}

$\mathrm{IC}_{50}$ values of $\mathrm{AmB}$ on intracellular amastigotes determined by luciferase assay. Mean $\pm S D(n=3)$.

${ }^{*} \mathrm{p}<0.001$ when compared with free AmB.

AmB: Amphotericin B; BMM- $\varphi$ : Bone marrow-derived macrophages; MN: Mannan; PLGA: Poly(o,t-lactide-co-glycolide); SD: Standard deviation. 


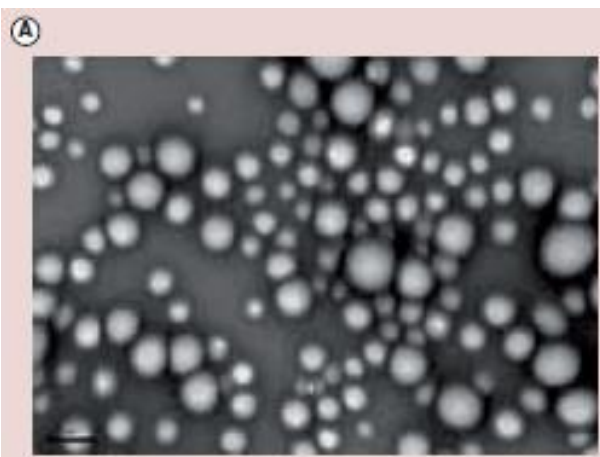

(c)

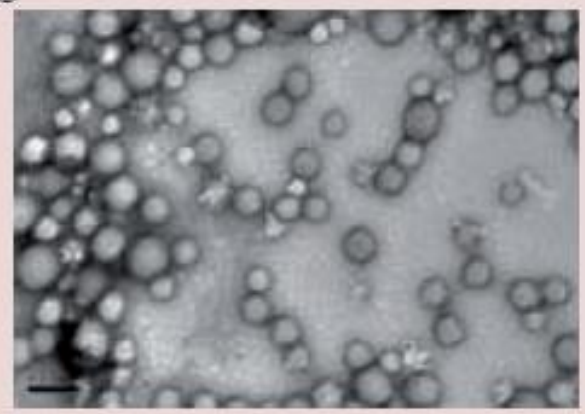

(E)

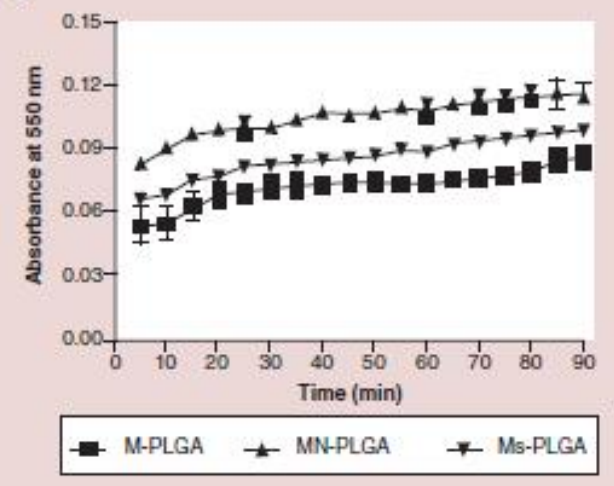

(B)

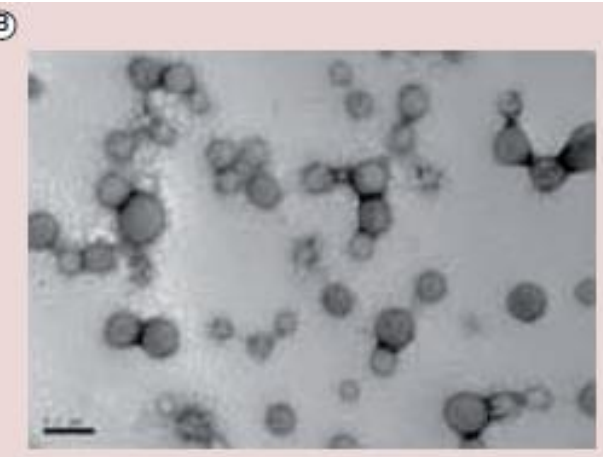

(D)

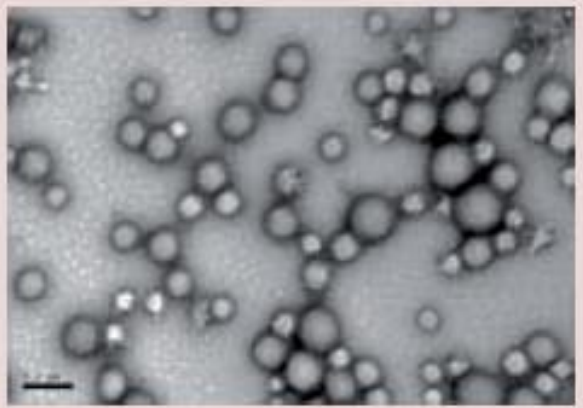

(๑)

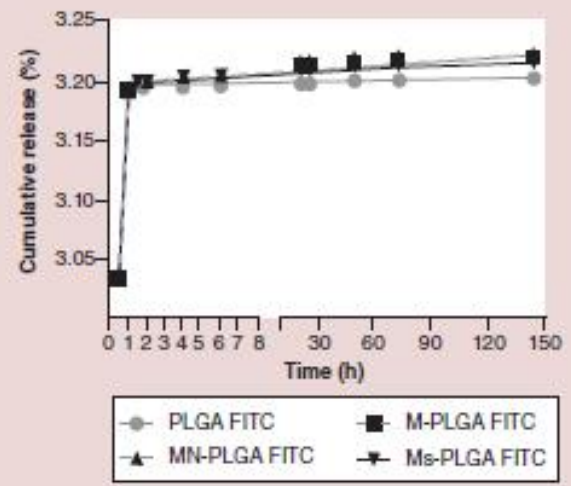

Figure 1. FITC-loaded poly(d,I-lactide-co-glycolide) nanospheres characterization. Morphology of nonfunctionalized (A) and functionalized M (B), MN (C), Ms (D) PLGA nanospheres at 80,000× magnifications; bars represent $0.2 \mu \mathrm{m}$. (E) Agglutination assay of M-, MN- and Ms- functionalized PLGA nanospheres with ConA. (F) FITC in vitro release kinetics from nonfunctionalized (PLGA FITC) and functionalized (M, MN, Ms) PLGA nanospheres. Mean \pm SDs ( $n$ =3). FITC: Fluorescein isothiocyanate isomer I; M: Mannose; MN: Mannan; Ms: Mannosamine; PLGA: Poly(d,Ilactide-co-glycolide); SD: Standard deviation. 


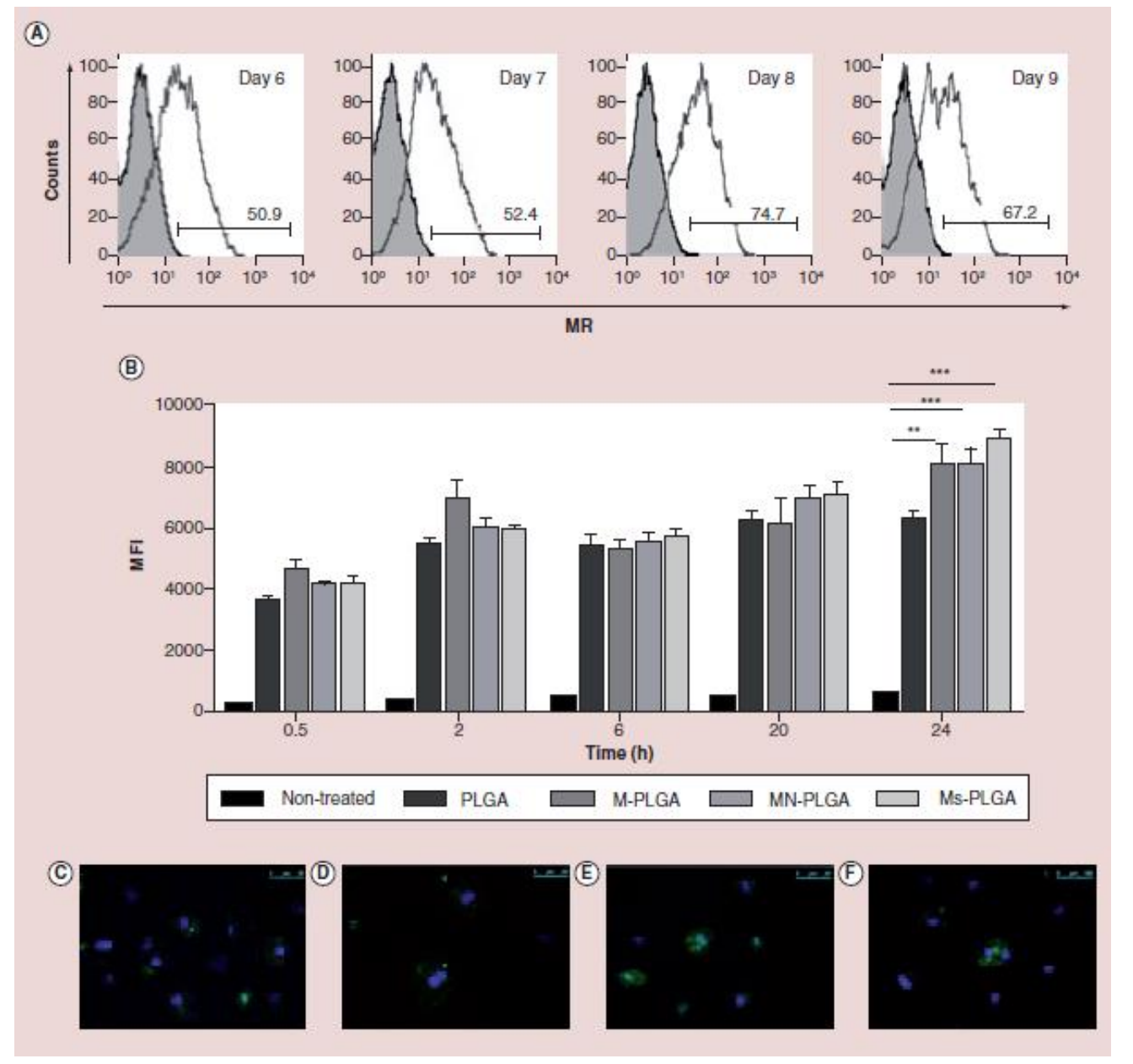

Figure 2. Mannose receptor expression during murine bone marrow-derived macrophage differentiation and cellular uptake of FITC-loaded nonfunctionalized and functionalized poly(d,I-lactide-co-glycolide) nanospheres. (A) Histograms representing MR expression at days 6, 7, 8 and 9 of differentiation. The solid gray and black lines represent F4/80+ unstained cells and F4/80+/MR+ cells, respectively. (B) Uptake of FITC-loaded nanospheres by bone marrow-derived macrophages with 8 days of differentiation at different incubation times, evaluated by flow cytometry. Bars represent mean $\pm S D(n=3)$. Fluorescence microscopy images of the internalization of FITC-loaded nonfunctionalized $(C)$ and $M(D), M N(E)$, Ms $(F)$ functionalized nanospheres by bone marrow-derived macrophages after $24 \mathrm{~h}$ of incubation. M: Mannose; MFI: Mean fluorescence intensity; MN: Mannan; MR: Mannose receptor; Ms: Mannosamine; PLGA: Poly(d,I-lactic-co-glycolic acid); SD: Standard deviation. 


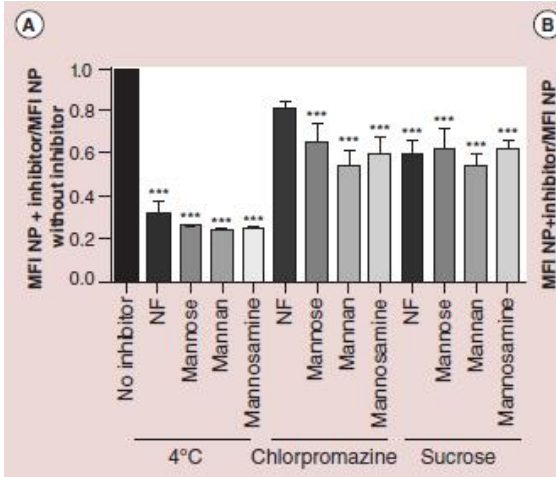

(D)

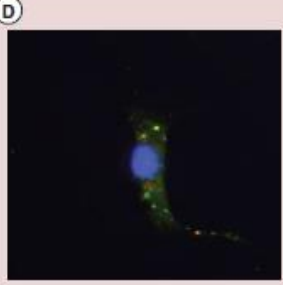

(B)

(E)

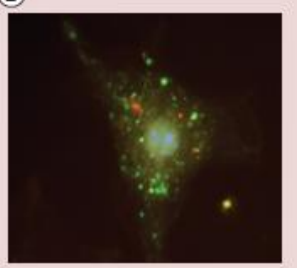

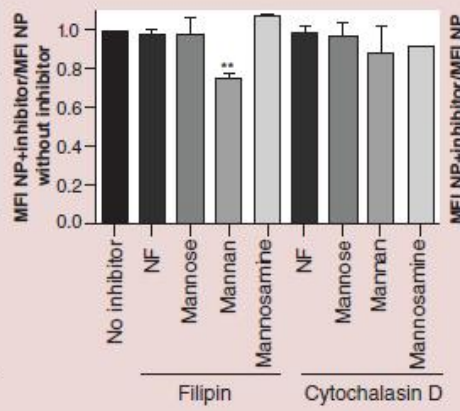

(F)

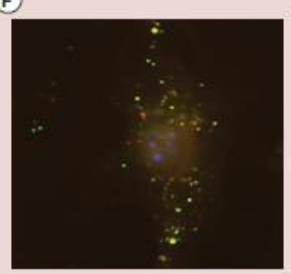

C)

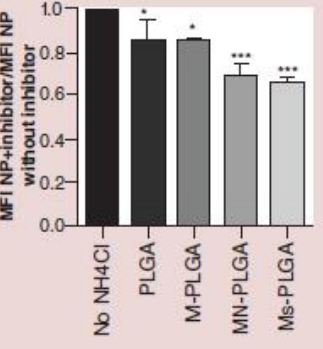

(G)

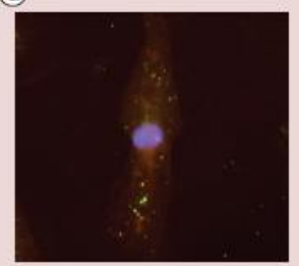

Figure 3. Mechanism of intracellular internalization of functionalized poly(d,I-lactide-co-glycolide) nanospheres. Effect of different inhibitors on the internalization of nonfunctionalized and functionalized PLGA nanospheres, (A) Inhibitors of clathrin-mediate endocytosis $\left(4^{\circ} \mathrm{C}\right.$; chlorpromazine and sucrose); (B) Filipin inhibits caveolae-mediated endocytosis and Cytochalasin $\mathrm{D}$ inhibits macropinocytosis. (C) Effect of $\mathrm{NH}_{4} \mathrm{Cl}$ on the internalization of nonfunctionalized and functionalized PLGA nanospheres. The results were normalized against the cells with no inhibitor, mean $\pm \mathrm{SD}(\mathrm{n}=3)$. ${ }^{*} \mathrm{p}<0.05,{ }^{* *} \mathrm{p}<0.01,{ }^{* * *} \mathrm{p}<0.001$ compared with cells with no inhibitor. Co-localization (yellow color) with lysosome of (D) nonfunctionalized and functionalized ([E] mannose; [F] mannan; [G] mannosamine), on bone marrow-derived macrophages. Nucleus is stained with DAPI (blue), lysossome with LysoTracker (red) and nanospheres shown in green fluorescence. Representative images are shown with a magnification of 1000x. M: Mannose; MFI: Mean fluorescence intensity; MN: Mannan; MR: Mannose receptor; Ms: Mannosamine; NF: Nonfunctionalized; NP: Nanoparticle; PLGA: Poly(d,I-lactide-co-glycolide); SD: Standard deviation. 


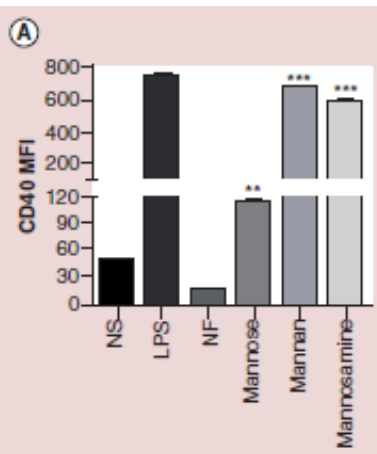

(D)

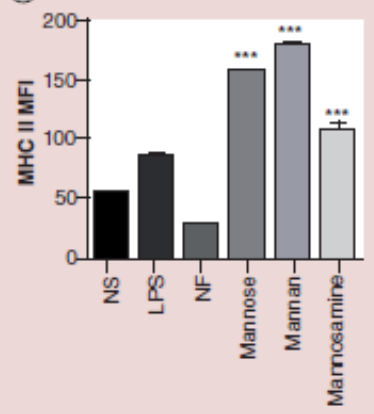

(ब)

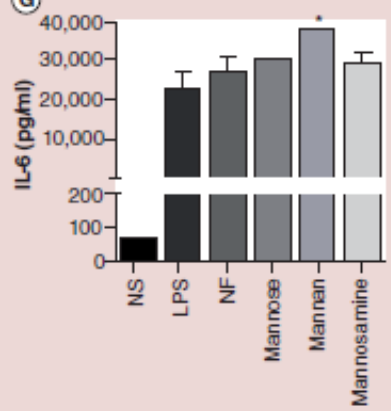

(B)

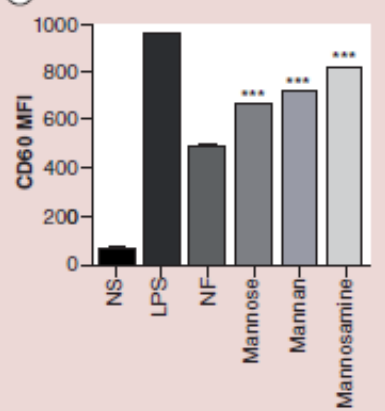

(E)

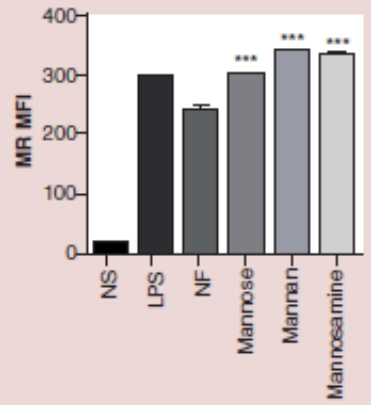

(1)

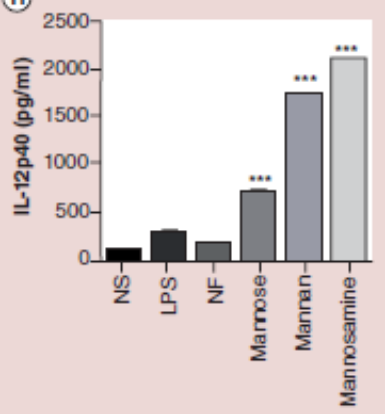

(C)

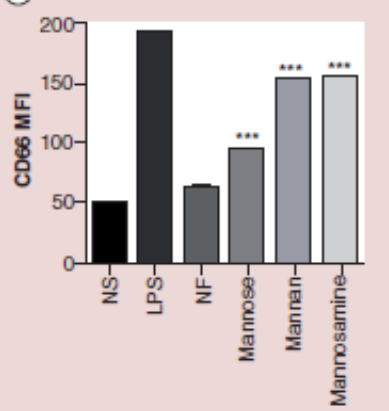

(F)

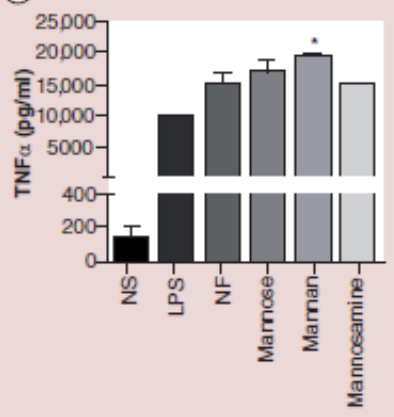

(1)

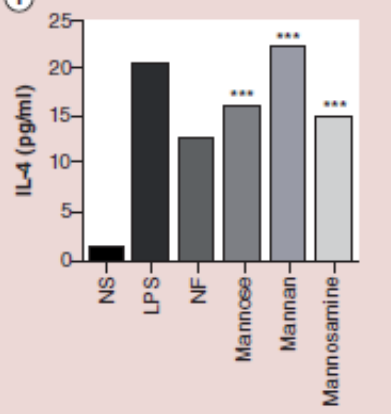

Figure 4. Immune-modulation of bone marrow-derived macrophages by functionalized poly(d,I-lactide-co-glycolide) nanospheres. Surface expression of (A) CD40, (B) CD80, (C) CD86, (D) MHCIl and (E) MR in BMM- $\varphi$ after $20 \mathrm{~h}$ of incubation. Profile of secreted cytokines induced in BMM- $\varphi$ by functionalized poly(d,l-lactide-co-glycolide) nanospheres (F) TNF-a, (G) IL- 6, (H) IL-12p40 and (I) IL- 4. NS and lipopolysaccharide-stimulated cells were used as negative and positive control, respectively. BMM- $\varphi$ were cultured with NF and functionalized (mannose, mannan and mannosamine) poly(d,I-lactide-co-glycolide) nanospheres. Results are expressed as the mean \pm SD of three independent experiments performed in duplicate. ${ }^{*} p<0.05$, ${ }^{* *} p<0.01$ and ${ }^{* * *} p<0.001$ compared with nonfunctionalized. BMM- $\varphi$ : Bone marrow-derived macrophages; LPS: Lipopolysaccharide; MFI: Mean fluorescence intensity; NF: Nonfunctionalized; NS: Nonstimulated; SD: Standard deviation. 

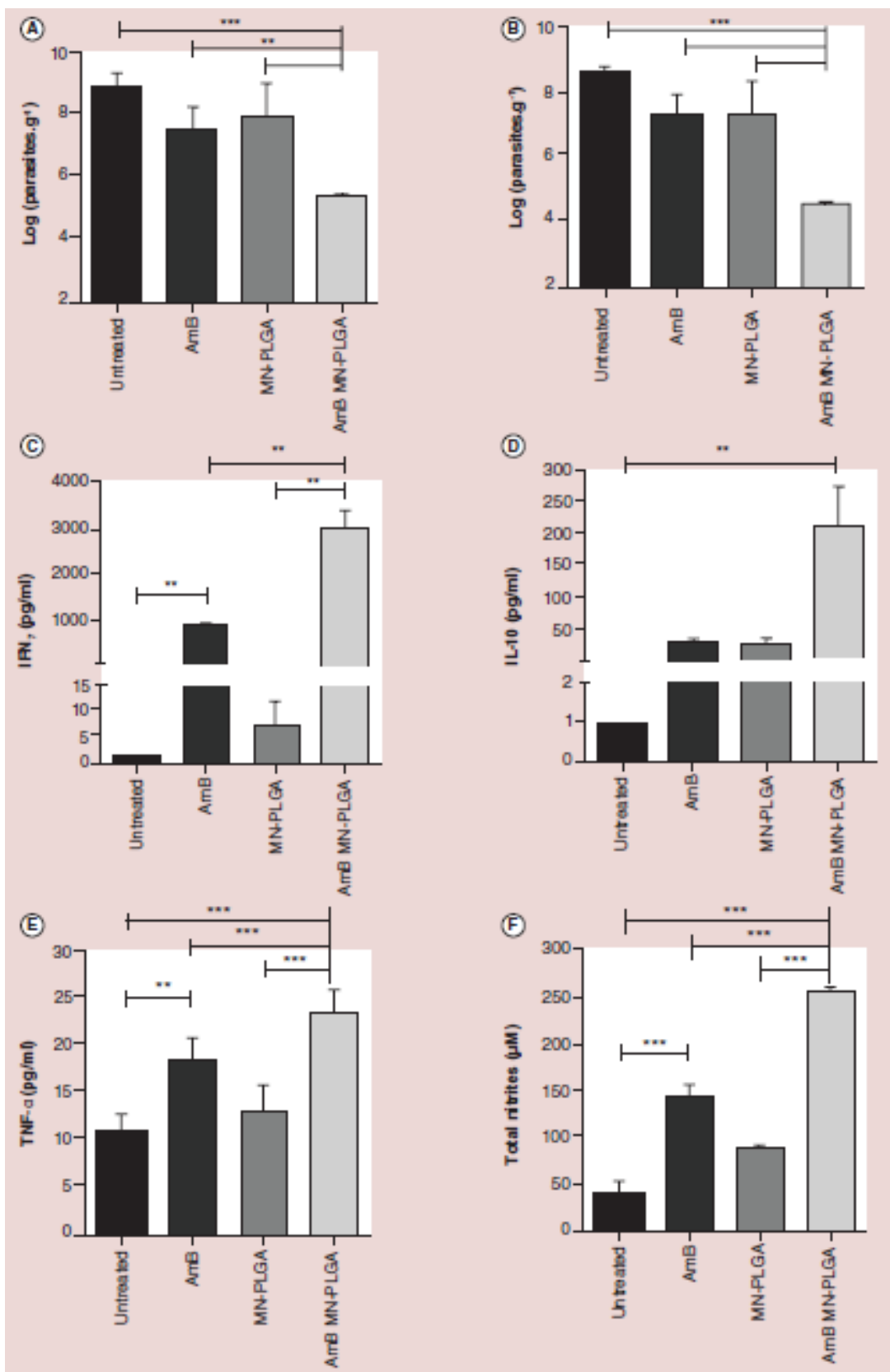

Figure 5. In vivo efficacy of AmB-loaded mannan-functionalized poly(d,I-lactide-co-glycolide) nanospheres in Leishmania-infected Balb/c mice (see facing page). One week after treatment administration, parasite burden was evaluated by limiting dilution assay in spleen (A) and liver (B). Profile of secreted cytokines IFN-Y (C), IL10 (D), TNF$\alpha(E)$ and nitric oxide production $(F)$ by spleen cells isolated from differently treated Leishmania infantum-infected Balb/c mice, after stimulation with soluble Leishmania antigens for $96 \mathrm{~h}$. Data represent mean \pm SD of a representative experiment from three carried out independently. ${ }^{*} p<0.05 ;{ }^{* *} p<0.01 ;{ }^{* * *} p<0.001$ when compared with the control group. AmB: Amphotericin B; MN: Mannan; PLGA: Poly(d,I-lactide-co-glycolide); SD: Standard deviation. 